\title{
Neonatal Determinants of Inadequate Breastfeeding: A Survey among a Group of Neonate Infants in Yaoundé, Cameroon
}

\author{
Ngwanou Dany Hermann, Georges Pius Kamsu Moyo* \\ Faculty of Medicine and Biomedical Sciences, University of Yaoundé 1, Yaoundé, Cameroon \\ Email: *kamsuzicfried@yahoo.fr, danyher2005@yahoo.com
}

How to cite this paper: Hermann, N.D. and Moyo, G.P.K. (2020) Neonatal Determinants of Inadequate Breastfeeding: A Survey among a Group of Neonate Infants in Yaoundé, Cameroon. Open Access Library Journal, 7: e6541.

https://doi.org/10.4236/oalib.1106541

Received: June 20, 2020

Accepted: July 17, 2020

Published: July 20, 2020

Copyright $\odot 2020$ by author(s) and Open Access Library Inc.

This work is licensed under the Creative Commons Attribution International License (CC BY 4.0).

http://creativecommons.org/licenses/by/4.0/

\begin{abstract}
Exclusive breastfeeding is essential for the newborn's development. According to the WHO, breastfeeding after childbirth should be initiated within the first 30 minutes to an hour following delivery. Early and adequate breastfeeding practices contribute to the reduction of neonatal morbidity and mortality, but are oftentimes not respected. The objective of this survey was to determine neonatal factors associated with breastfeeding initiation and its adequate practice. We carried out a cross-sectional study at the Yaoundé Gynaeco-Obstetric and Paediatric Hospital from December 2018 to May 2019. All livebirth infants weighing $>2000 \mathrm{~g}$ were included. A total of 250 neonate infants were enrolled. Newborns had satisfactory adaptation to extra-uterine life in 240 cases (96\%), and vaginal route was the main mode of delivery in 172 women (70\%). The newborns had a mean gestational age (GA) of $38.4 \pm 1.6$ weeks and a mean birth weight of $3168.6 \pm 508.7 \mathrm{~g}$. The male sex predominated by a ratio of 1.29 . The average time of breastfeeding initiation was 120 minutes. Only $100(40 \%)$ newborns were put to the breast within the first hour after birth and up to 153 (61.2\%) mothers practiced inadequate breastfeeding. Among factors associated with inadequate breastfeeding practices, neonatal determinants were neonates delivered through caesarean section, gestational age $(\mathrm{GA})<37$ weeks, low birth weight $(\mathrm{LBW})<$ $2500 \mathrm{~g}$ and neonatal infection. Therefore, a number of neonatal factors may disrupt breastfeeding in newly delivered women. These may be prevented by reducing the rate of caesarean and premature deliveries through efficient antenatal follow-up, the prevention of risk factors to neonatal sepsis during the perinatal period.
\end{abstract}

\section{Subject Areas}

Gynecology \& Obstetrics 


\section{Keywords}

Breastfeeding, Neonatal Factors, Cameroon

\section{Introduction}

Breastfeeding is a natural process required for the growth of neonate infants. Indeed, the breastmilk contains essential growth and immunological factors which are necessary for the neonate's development [1]. Colostrum is considered as the first vaccine, perfect food for the newborn as well and is recommended within 30 minutes to one hour after birth [1]. The Early Initiation of breastfeeding (EIBF) has several benefits, including the prevention of hypoglycemia and hypothermia. On the other hand, it favors the early release of meconium, thus contributing to a certain measure to the prevention of neonatal jaundice. There is substantial evidence that breastfeeding decreases neonatal mortality, morbidity, sepsis-related deaths, diarrhea and respiratory infections in neonates and infants under 5 years [2] [3]. Furthermore, from a sociocultural point of view, larger infants are thought to be the healthiest in a number of African traditions, motiving women to breastfeed. Nevertheless, protective effects against obesity and other chronic diseases in the long-term have been reported in adequately breastfed infants [2] [3]. A number of research studies worldwide advocate the fact that breastfeeding largely contributed to the reduction of about 804.000 deaths (11.6\%) among under-five children in 2011 [4] [5]. During the same period, countries such as India, had made it mandatory to keep the mother and baby at least 48 hours in the hospital after delivery [6]. This led to the enhancement of breastfeeding practices under the watchful monitoring of the health staff. In effect, it has been shown that medical staff and family support are major cues to early initiation of breastfeeding [7] [8] [9]. In Cameroon, according to the Demographic and Health Survey conducted in 2018, 39.7\% of infants under 5 months of age were described as exclusively breastfed, though no further information about the time of breastfeeding initiation was given [10]. However, a number of determinants including neonatal-related factors may be associated with inadequate breastfeeding practices. This study had as main objective to identify neonatal determinants of inadequate breastfeeding practices among a group of neonates in Yaoundé, the capital city of Cameroon.

\section{Methodology}

We conducted a cross-sectional and analytical study over a six-month period from December 2018 to May 2019. The survey took place at the Yaoundé Gynaeco-Obstetric and Paediatric Hospital, which is a University Teaching Hospital in Cameroon. We included all livebirth infants weighing more than 2000 g, with no initial contraindication to breastfeeding and whose mothers consented to participate in our study. Newborns having congenital digestive 
malformations preventing breastfeeding were excluded. The recruitment was consecutive and exhaustive. The enrolled neonates were observed during the first week after delivery to detect those that would be adequately breastfed in terms of timely initiation of the process, the effectiveness of the act, exclusiveness and duration, in conformity with WHO's recommendations. Sampling was consecutive. The sources of information were mothers and medical records. A pretested questionnaire was administered and the relevant neonatal-related variables sought. Data were recorded and analyzed using CS Pro version 6.2 and SPSS version 20.0. Chi-square testing was used to establish statistical associations between the variables. The $\mathrm{P}$ value $<0.05$ indicated the statistical significance of the associations established. The Odds ratio with $95 \%$ confidence interval was used to identify the risk relationships.

The assessment of breastfeeding practices was based on four characteristics including timely initiation within an hour following birth, exclusiveness through the use of breastmilk substitute or not, the effectiveness of the breastfeeding technique and the duration based on the intention to breastfeed the infant or not during the first six months.

Ethical clearances from the Institutional Ethics and Research Committee of the Faculty of Medicine and Biomedical Sciences of the University of Yaoundé 1 and the Yaoundé Gynaeco-Obstetric and Paediatric Hospital were obtained before the beginning of the survey. The data collected was kept strictly confidential and used only for the purposes of the study.

\section{Results}

\subsection{Perinatal Characteristics}

We enrolled 250 women who met the inclusion criteria during the study period. The mean gestational age (GA) was $38.4 \pm 1.6$ weeks; the average birth weight was $3168.6 \pm 508.7 \mathrm{~g}$. Vaginal delivery was the main route of childbirth and 240 (96\%) newborns had a good adaptation to extra-uterine life. The immediate neonatal period was in general uncomplicated though 60 (24\%) newborns were admitted to the neonatology unit for one reason or the other as shown in Table 1.

\subsection{Inadequate Breastfeeding Practices}

The average time to start breastfeeding after delivery in our series was 120 minutes ( 2 hours). Delayed initiation of breastfeeding was reported in all neonates with inadequate breastfeeding, while 102 neonates (66.8\%) received breastmilk substitutes. Poor breastfeeding techniques were practiced by 77 women (50.3\%), while 51 (33.3\%) women among those with inadequate breastfeeding practices had no intention to exclusively breastfeed during the first six months following the child's birth.

\subsection{Neonatal Determinants of Inadequate Breastfeeding}

Caesarean delivery, gestational age (GA) below 37 weeks of pregnancy, low 
Table 1. Perinatal characteristics.

\begin{tabular}{|c|c|c|}
\hline Variables & $\mathrm{N}(250)$ & Percentages (\%) \\
\hline \multicolumn{3}{|l|}{ Mode of delivery } \\
\hline Vaginal route & 172 & 68.8 \\
\hline Caesarean section & 78 & 31.2 \\
\hline \multicolumn{3}{|l|}{ Post-Partum } \\
\hline Simple & 230 & 92 \\
\hline Complicated & 20 & 8 \\
\hline \multicolumn{3}{|l|}{ Gestational age } \\
\hline$<37$ & 21 & 8.4 \\
\hline$[37-42[$ & 222 & 88.8 \\
\hline$\geq 42$ & 7 & 2.8 \\
\hline \multicolumn{3}{|l|}{ Birth weight } \\
\hline$<2500 \mathrm{~g}$ & 18 & 7.2 \\
\hline$[2500-4000]$ & 216 & 86.4 \\
\hline$\geq 4000 \mathrm{~g}$ & 16 & 6.4 \\
\hline \multicolumn{3}{|l|}{ Diagnosis on admission } \\
\hline Risk of infection & 15 & 25 \\
\hline Neonatal infection & 14 & 23.3 \\
\hline Neonatal jaundice & 14 & 23.3 \\
\hline Mild birth asphyxia & 4 & 6.7 \\
\hline Other & 13 & 21.7 \\
\hline
\end{tabular}

birthweight and neonatal infection at birth were neonatal factors associated with inadequate breastfeeding practices after bivariate analysis as shown in Table 2.

Table 2. Neonatal factors associated with inadequate breastfeeding.

\begin{tabular}{cccccc}
\hline \multirow{2}{*}{ Variables } & \multicolumn{2}{c}{ Breastfeeding practice } & & \multirow{2}{*}{ OR } & p-Value \\
\cline { 2 - 3 } & Inadequate (\%) & Adequate (\%) & & \\
\hline Caesarean delivery & $72(92.3)$ & $6(7.7)$ & 13.5 & $<0.001$ \\
Gestational age < 37 weeks & $18(85.7)$ & $3(14.3)$ & 4.2 & 0.016 \\
Low birthweight < 2500 g & $15(83.3)$ & $3(16.7)$ & 3.4 & 0.045 \\
Neonatal infection & $13(92.9)$ & $1(7.1)$ & 10.9 & 0.009 \\
\hline
\end{tabular}

\section{Discussion}

The assessment of the breastfeeding practices was mainly based on four characteristics including the timely initiation of the process, the use of breastmilk substitute such as formula milk, water or sugar solution, the effectiveness of the breastfeeding technique and the duration. Amongst these various descriptions, delayed breastfeeding initiation was the most occurring in $97 \%$ of women with 
inadequate breastfeeding practices. This induced the use of breastmilk substitutes in over $86 \%$, and more than $80 \%$ not having the intention to do exclusive breastfeeding over 6 months. The rate of Early Initiation of Breastfeeding (EIBF) in our survey was $40 \%$, which is quite lower than values obtained in the majority of developed countries such as Australia, where values as high as $98 \%$ have been reported [11]. This is also lower than incidences reported in a number of African developing countries such as Ethiopia (73.1\%), even though our sample size was relatively inferior [12] [13] [14] [15]. The effectiveness of the act of breastfeeding included deep, tonic and slow suctions separated or not by short pauses and yielding breastmilk into the baby's mouth. The duration was assessed in terms of intention to breastfeed exclusively during the first six months following the child's birth. This was due to the difficulty to follow all women over a six-month period. However, this assumption may involve some selection bias, though breastfeeding planning may largely contribute to its implementation. A number of research works have reveal associations between breastfeeding initiation and exclusive breastfeeding within recommended duration [16] [17]. In effect, there is strong evidence that babies with delayed breastfeeding initiation, mainly due to caesarean delivery are likely to be fed with formula milk within the first three days of life, and are as well less susceptible to be exclusively breastfed during the first six months [16] [17]. Therefore, low rates of EIBF may be responsible for reduced exclusive breastfeeding and hence inadequate breastfeeding practices.

Delivery by caesarean section is a known predictive factor for inadequate breastfeeding practices, mainly due to considerable delayed initiation of the process. In effect, there are considerable hormone variations after caesarean delivery involving reduced blood levels of prolactin, oxytocin and endorphin, which are however necessary for breastmilk production, ejection and mother-infant attachment [18]. Moreover, general anesthesia, post-operative pains and the use of opioid analgesics in the mother may be responsible for neonatal impregnation and sedation with these drugs through placenta blood or breastfeeding, causing altered consciousness and hence delayed initiation of Breastfeeding during the immediate postpartum [19] [20]. Furthermore, neonates with elective caesarean delivery encounter less stress, little cortisol secretion and poor lung maturation [21]. They may thus develop transient tachypnea which causes respiratory distress resulting in delayed breastfeeding initiation and the use of breastmilk substitutes [21]. It has been described that newborns which have gone through labor, and delivered by vaginal route develop more appetite and improved suckling reflexes than others [22]. More so, indications of emergency caesarean delivery in close to $50 \%$ of cases may be associated with acute fetal distress which could yield ill neonates, unfit to breastfeed. As such, the mother and newborn separation for more attention and care, is a common practice immediately after caesarean delivery, but with undesired disruption of breastfeeding practices [3].

Though there is evidence that breastmilk feeding reduces mortality, short and long-term morbidity in high risk infants including those with infection, prema- 
turity, and low birthweight, a delay in initiating breastfeeding and inadequate breastmilk feeding is often observed [1] [2] [3]. This is due to the fact that such newborns may be delivered with immediate neonatal emergencies such as neurologic, infectious, thermal regulation, digestive and feeding disorders. In fact, a neonatal emergency may be considered as a potentially life-threatening disorder or anomaly which occurs within the first 28 days after birth [23]. In a cross-sectional study to determine neonatal emergencies in our context, a prospective recruitment of neonates showed that $88 \%$ of all neonatal emergencies were medical or non-surgical, and more than $5 \%$ neonates had mixed emergencies. These emergencies are predominated by neonatal infections or sepsis, prematurity, birth asphyxia and jaundice worldwide. In more than $50 \%$ of cases, they occur within the first week of life, and the related mortality rate may be as high as $5 \%$ in our context [23]. There may exist a vicious cycle between breastmilk feeding and neonatal illness, given that the absence of breastfeeding or an inadequate breastmilk feeding could be responsible for illness and complications in newborns, which in turn alters the feeding process and vice versa. The acknowledgement of this relationship justifies the promotion of early breastfeeding initiation for immunological and nutritional purposes.

Prematurity may be considered as the delivery of a neonate infant beyond 22 and before 37 completed weeks of gestation. Preterm infants are generally characterized by a low-birth weight between $500 \mathrm{~g}-2400 \mathrm{~g}$ which may be associated with hypotrophy in some cases. Nevertheless, normal size or eutrophy, as well as macrosomia with respect to gestational age, may also occur among preterm infants, though rare. However, premature infants born too early usually lack sufficient biological equipment to cope with various life necessities and exigencies immediately after birth, thus requiring appropriate assistance. Among various deficiencies, these infants present with weak neonatal suckling reflex and low enzyme secretion including amylases, peptidases, and lipases, due to hepatic, pancreatic and intestinal immaturity among others [24]. These are responsible for ingestion, digestion and absorption anomalies associated with inadequate breastfeeding. Moreover, necrotizing enterocolitis (NEC) is a particular intestinal disorder of the preterm infant which may be associated with poor breastmilk feeding. Though its overall incidence has been reducing steadily during the past few years, NEC has been recognized for decades as the most common life-threatening medico-surgical intestinal emergency in newborns. The disease might be associated with short and long-term complications, prolong hospitalization, and be cost worthy. Nevertheless, primary prevention by breastfeeding promotion is pertinent in improving neonatal outcome.

Some limitations to this survey may include the sample size which was relatively smaller compared to similar studies conducted in other contexts. The study was circumscribed to a given group at a particular time and so results may only be specific to the said group. Whereas, a calculated, and/or a larger sample size adds more power and significance to a survey, with the possibility of trans- 
posing results to the general population with considerable reliability. More so, in considering the duration of six months exclusive breastfeeding, assimilating the 'intension to do so', with the act might involve some selection, analysis and eventually interpretation bias. A longitudinal study may have enabled to identify more determinants due to better elaborated prospective or retrospective 'follow-up' of subjects' characteristics.

\section{Conclusion}

From this survey, it appears that neonatal determinants of delayed breastfeeding initiation and inadequate breastfeeding practices are mainly neonatal emergencies, characterized by life-threatening conditions due to caesarean delivery, preterm, low birth weight and neonatal infection. In effect, in such cases feeding may be delayed in order to rapidly attend to vital emergencies thereby causing retardation of breastfeeding initiation and overall disrupted breastmilk feeding process. This may be prevented by promoting efficient antenatal follow-up, with the detection and resolution of maternal and fetal problems, the improvement of vaginal delivery practices and the restriction of breastfeeding contraindications to strict absolutes.

\section{Acknowledgements}

Hospitals authorities, all collaborators to this project.

\section{Funding}

Private.

\section{Conflict of Interest}

The authors declare that they have no competing interests.

\section{References}

[1] WHO (2020) Breastfeeding. http://www.who.int/topics/breastfeeding/en

[2] Hess, C., Ofei, A. and Mincher, A. (2015) Breastfeeding and Childhood Obesity among African Americans: A Systematic Review. MCN: The American Journal of Maternall Child Nursing, 40, 313-319. https://doi.org/10.1097/NMC.0000000000000170

[3] Khan, J., Vesel, L., Bahl, R. and Martines, J. (2015) Timing of Breastfeeding Initiation and Exclusivity of Breastfeeding during the First Month of Life: Effects on Neonatal Mortality and Morbidity-A Systematic Review and Meta-Analysis. Maternal and Child Health Journal, 19, 468-479. https://doi.org/10.1007/s10995-014-1526-8

[4] Black, R., Victora, C., Walker, S., Bhutta, Z., Christian, P., de, Onis, M., et al. (2013) Maternal and Child Undernutrition and Overweight in Low-Income and Middle-Income Countries. The Lancet, 382, 427-451. https://doi.org/10.1016/S0140-6736(13)60937-X

[5] Quyen Thi-Tu, B., Hwa-Young, L., Thi-Kim, L., DoVan, D. and Lan ThiHoang, V. 
(2016) Trends and Determinants for Early Initiation of and Exclusive Breastfeeding under Six Months in Vietnam: Results from the Multiple Indicator Cluster Surveys, 2000-2011. Global Health Action, 9, Article 29433. https://doi.org/10.3402/gha.v9.29433

[6] MaJra, J. and ViJay, K. (2016) Barriers to Early Initiation and Continuation of Breastfeeding in a Tertiary Care Institute of Haryana: A Qualitative Study in Nursing Care Providers. Journal of Clinical and Diagnostic Research, 10, 16-20. https://doi.org/10.7860/JCDR/2016/19072.8559

[7] Setegn, T., Gerbaba, M. and Belachew, T. (2011) Determinants of Timely Initiation of Breastfeeding among Mothers in Goba Woreda, South East Ethiopia: A Cross Sectional Study. BMC Public Health, 11, Article No. 217.

https://doi.org/10.1186/1471-2458-11-217

[8] Kimani-Murage, E., Kyobutungi, C., Ezeh, A., Wekesah, F., Wanjohi, M., Muriuki, P., et al. (2013) Effectiveness of Personalized, Home-Based Nutritional Counselling on Infant Feeding Practices, Morbidity and Nutritional Outcomes among Infants in Infants in Nairobi Slums: Study Protocol for a Cluster Randomised Controlled Trial. Trials, 14, 445. https://doi.org/10.1186/1745-6215-14-445

[9] Adugna, D. (2014) Women's Perception and Risk Factors for Delayed Initiation of Breastfeeding in Arba Minch Zuria, Southern Ethiopia. International Breastfeeding Journal, 9, 8. https://doi.org/10.1186/1746-4358-9-8

[10] Institut National de la Statistique (INS), et ICF (2019) Enquête Démographique et de Santé du Cameroun 2018. Indicateurs Clés. INS et ICF, Yaoundé, Rockville, Report No. 5.

[11] Federal Ministry of Health (2010) Health Sector Development Program IV Woreda Based Annual Core Plan. Australia. Report No. 2.

[12] Bimerew, A., Teshome, M. and Kassa, G. (2016) Prevalence of Timely Breastfeeding Initiation and Associated Factors in Dembecha District, North West Ethiopia: A Cross-Sectional Study. International Breastfeeding Journal, 11, 28. https://doi.org/10.1186/s13006-016-0087-4

[13] Wolde, T., Birhanu, T. and Ejeta I. (2014) Prevalence and Determinants of Timely Initiation of Breastfeeding among Lactating Mothers of Urban Dwellers in Western Ethiopia. Food Science and Quality Management, 31, 2225-2557.

[14] McLachlan, H. and Forster, D. (2016) Initial Breastfeeding Attitudes and Practices of Women Born in Turkey, Vietnam and Australia after Giving Birth in Australia. International Breastfeeding Journal, 1, 7. https://doi.org/10.1186/1746-4358-1-7

[15] Amin, T., Hablasa, H. and Qader, A. (2010) Determinants of Initiation and Exclusivity of Breastfeeding in Al Hassa, Saudi Arabia. International Nursing Review, 6, 59-68. https://doi.org/10.1089/bfm.2010.0018

[16] Kuyper, E., Vitta, B. and Dewey, K. (2014) Implications of Cesarean Delivery for Breastfeeding Outcomes and Strategies to Support Breastfeeding. A\&T Technical Brief, No. 8, 1-9.

[17] Constance, A., Gewa, M., Monica, O. and Lauren, S. (2011) Determinants of Early Child-Feeding Practices among HIV-Infected and Non Infected Mothers in Rural Kenya. Journal of Human Lactation, 27, 239-249. https://doi.org/10.1177/0890334411403930

[18] Odent, M. (2005) Césariennes:questions, effets, enjeux. Alerte face à la banalisation. Le Souffle d'Or. Elsevier Masson, Barret-sur-Méouge, 200 p.

[19] Beilin, Y., Bodian, C., Weiser, J., Hossain, S., Arnold, I. and Feierman, D. (2005) Effect of Labor Epidural Analgesia with and without Fentanyl on Infant Breast-Feeding: A Pro- 
spective, Randomized, Double-Blind Study. Anesthesiology, 103, 1211-1217. https://doi.org/10.1097/00000542-200512000-00016

[20] Baumgarder, D., Muehl, P., Fischer, M. and Pribbenow, B. (2003) Effect of Labor Epidural Anesthesia on Breast-Feeding of Healthy Full-Term Newborns Delivered Vaginally. The Journal of the American Board of Family Practice, 16, 7-13. https://doi.org/10.3122/jabfm.16.1.7

[21] Moyo, G.P.K. and Tetsiguia, J.R.M. (2020) Discussing the "First Cry" as an Initial Assessment for Neonates. American Journal of Pediatrics, 6, 129-132. https://doi.org/10.11648/j.ajp.20200602.22

[22] Hyde, M.J., Mostyn, A., Modi, N. and Kemp, P.R. (2012) The Health Implications of Birth by Caesarean Section. Biological Reviews of the Cambridge Philosophical Society, 87, 229-243. https://doi.org/10.1111/j.1469-185X.2011.00195.X

[23] Moyo, G.P.K., Sobguemezing, D. and Adjifack, H.T. (2020) Neonatal Emergencies in Full-Term Infants: A Seasonal Description in a Pediatric Referral Hospital of Yaoundé, Cameroon. American Journal of Pediatrics, 6, 87-90. https://doi.org/10.11648/j.ajp.20200602.13

[24] Moyo, G.P.K., Nguedjam, M. and Miaffo, L. (2020) Necrotizing Enterocolitis Complicating Sepsis in a Late Preterm Cameroonian Infant. American Journal of Pediatrics, 6, 83-86. https://doi.org/10.11648/j.ajp.20200602.12 